Médecine à la frontière : le recours aux professionnels de santé afghans en contexte d'urgence humanitaire

\title{
Chloé Tisserand
}

\section{OpenEdition}

\section{Journals}

Édition électronique

URL : http://journals.openedition.org/rfst/405

DOI : $10.4000 /$ rfst.405

ISSN : 2492-3672

Éditeur

Espaces et SOciétés (UMR 6590)

Référence électronique

Chloé Tisserand, « Médecine à la frontière : le recours aux professionnels de santé afghans en contexte d'urgence humanitaire », Revue francophone sur la santé et les territoires [En ligne], Les circulations en santé : des produits, des savoirs, des personnes en mouvement, mis en ligne le 16 décembre 2019, consulté le 06 avril 2021. URL : http://journals.openedition.org/rfst/405 ; DOI : https://doi.org/10.4000/rfst.405

Ce document a été généré automatiquement le 6 avril 2021.

\section{cc) (i) $(9)$}

La Revue francophone sur la santé et les territoires est mise à disposition selon les termes de la Licence Creative Commons Attribution - Pas d'Utilisation Commerciale - Partage dans les Mêmes Conditions 4.0 International. 


\title{
Médecine à la frontière : le recours aux professionnels de santé afghans en contexte d'urgence humanitaire
}

\author{
Chloé Tisserand
}

\section{Introduction}

1 Les frontières se multiplient en Europe et dans le monde (Vallet, 2014) suite aux craintes d'une grande partie des gouvernements de voir déferler les exilés sur le continent (Smith, 2018) et d'un sentiment d'insécurité généralisé. Mais les travaux scientifiques ont démontré que cette peur est infondée et qu'il est préférable de parler de crise européenne de l'accueil plutôt que de crise des réfugiés (Héran, 2016). Cette crise de l'accueil se traduit par la création d'espaces de transit appelés «lieuxfrontières » (Cuttitta, 2015) avec une population laissée à la marge, qui évolue dans un monde de camps, le "couloir des exilés" (Agier, 2011). En attendant de pouvoir traverser la frontière franco-anglaise, ces personnes originaires d'Irak, d'Afghanistan, de la Corne de l'Afrique connaissent l'errance qui se réduit à vivre une vie dans un milieu hostile, rythmée par les violences réelles et symboliques (Guenebeaud, 2017), par la dépendance à l'égard des associations et à subir des conditions de vie insupportables qui se répercutent sur leur état de santé. À Calais, cette situation dure depuis les années 1990. Par conséquent, l'hôpital, l'un des rares lieux d'accueil institutionnel pour ces populations, a, au fil de cette expérience, repensé son organisation de travail pour prendre en compte, dans la prise en charge des soins des exilés, les « effets-frontières ». Son service, la permanence d'accès aux soins de santé (PASS-CH) ${ }^{1}$ qui s'adresse à toute personne dépourvue de couverture sociale, en est l'illustration et ce dispositif s'est retrouvé en première ligne pour gérer l'urgence humanitaire. Ce modulaire, dit PASS fixe, situé à côté du corps principal de l'hôpital et à proximité des urgences accueille les blessés à la frontière, ceux qui échouent dans leurs tentatives de passage vers l'Angleterre (sections de doigts dues aux accrochages contre les barbelés, fractures liées aux chutes de camions ou trains), les malades atteints de pathologies de l'errance 
(la gale, la tuberculose, les maladies saisonnières). La PASS-CH propose une prise en charge médico-sociale qui prend la forme d'un travail d'équipe entre l'assistante sociale et le personnel médical et paramédical.

L'urgence humanitaire, c'est aussi la gestion des flux migratoires avec un nombre de patients qui varie chaque jour, d'un mois à l'autre. Alors dans son format la PASS-CH, comme un mouvement d'accordéon, se déploie lorsque le nombre d'exilés est important sur le territoire et se resserre lorsqu'il diminue. Le fonctionnement de la PASS-CH s'appuie sur une équipe paramédicale et médicale composée d'une assistante sociale, d'infirmiers, d'une secrétaire et d'un interprète qui travaillent quotidiennement du lundi au vendredi dans le service; sur des vacations (rémunérées une centaine d'euros la journée) de médecins qui interviennent chaque jour ; sur des vacations dentaires et psychologiques. Puisqu'elle s'adapte aux flux migratoires, la PASS est polymorphe. Il faut distinguer trois périodes durant lesquelles les observations ont été réalisées.

D'abord, celle avant 2014 où la plupart des médecins français qui y intervenaient, étaient issus de cabinets libéraux et étaient mobilisés afin de renforcer l'absence de volontariat chez les professionnels hospitaliers.

Dans un second temps, la création de la «new jungle » à partir de 2014 a mis davantage en visibilité la crise de l'accueil. Ce surnom de «new jungle» a été donné par les bénévoles et repris par les exilés pour décrire ce que les autorités appelaient la Lande (Galitzine-Loumpet \& Moghani, 2018) à savoir un terrain situé en périphérie de ville sur lequel les exilés pouvaient s'installer sans être menacés d'expulsion. De 2014 à octobre 2016, jusqu'à 7500 exilés (chiffres préfecture) - 10000 (chiffres des associations) ont vécu sur ce terrain qualifié de " plus grand bidonville d'Europe » (La Voix du Nord, 2017 et Le Monde, 2016). Ainsi, l'hôpital a créé en plus de la PASS-CH, une antenne de la PASS - la PASS-Ferry -près de la «new-jungle » afin de trier «le tout venant » et d'orienter les patients atteints de maladies chroniques vers la PASS-CH ou vers les urgences ou spécialistes pour les cas les plus graves (Rodriguez \& Tisserand, 2017). L'hôpital a alors augmenté ses effectifs : on recense par exemple en 2013 en équivalent temps plein (ETP) 0,8 médecins contre 3 en 2016, 0,8 infirmiers contre 7,8 en 2016. Dans ce contexte, des soignants en situation de reclassement professionnel et des soignants exilés ont constitué un vivier sur lequel l'hôpital s'est appuyé pour trouver une solution rapide et répondre à l'urgence humanitaire. Des médecins afghans se sont donc greffés à l'équipe de soignants majoritairement française pour augmenter les effectifs.

5 Dans un dernier temps, l'après "new jungle " (octobre 2016) a laissé place à une réduction des effectifs suite à l'arrêt de la PASS-Ferry, avec un écartement des médecins libéraux et un maintien des médecins afghans à la PASS-CH. «Afghans » est un terme global qui désigne leur nationalité et permettent de les distinguer des professionnels français ici dans cet article mais il ne faut pas oublier derrière ce terme toutes les nuances et singularités liées à leur identité personnelle: deux d'entre eux indiquent être d'origine pachtoune, deux autres se disent tadjiks. Ces communautés d'appartenance ont leur histoire locale en Afghanistan qui comprend notamment des clivages communautaires (Dorronsoro, 1994). On pense au commandant Massoud, tadjik, tué par Al-Qaïda, organisation soutenue par des Pachtounes.

6 Les métamorphoses de la PASS favorisent donc la circulation des professionnels : en effet, les médecins libéraux croisent des médecins hospitaliers (français et afghans), des 
paramédicaux issus de services spécialisés (pneumologie, EHPAD, traumatologie, etc.) et qui se trouvent ou pas en situation de reclassement professionnel. Il existe aussi un turn over des soignants qui fait du dispositif médical un lieu potentiel de circulation des savoirs et des échanges. Ainsi cet article vise à souligner les altérations du travail médical qui ont pu se produire depuis l'intégration des médecins afghans au cœur d'une médecine dédiée aux précaires. En effet, nous verrons que cette inclusion dans le système de soin de santé constitue un atout pour les soignants français. Pour autant, l'appartenance culturelle des professionnels afghans peut aussi leur faire du tort puisque des clivages communautaires peuvent être réactivés dans la relation soignantsoigné. Mais nous verrons aussi que cette expérience à la PASS peut aussi servir les intérêts des soignants afghans qui cherchent à se reconstruire professionnellement.

7 Dans le cadre de ma thèse en sociologie qui vise surtout à comprendre ce que signifie de soigner les exilés en transit et d'essayer de définir les caractéristiques et spécificités de la médecine à la frontière, j'ai pris le parti d'aborder seulement le point de vue des soignants pour saisir ce que la rencontre avec leurs patients exilés produit sur eux d'un point de vue professionnel et émotionnel. J'ai réalisé depuis 2013 des observations non participantes jusqu'en 2019 et une trentaine d'entretiens auprès des professionnels de la PASS de Calais qui s'y sont succédé à différentes périodes entre 2006 et 2019. J'ai aussi $\mathrm{pu}$ observer des professionnels à la PASS de Dunkerque. Les soignants exilés ne représentent pas le cœur de mon sujet de thèse mais bien une sous-partie d'un de mes chapitres alors j'ai extrait les temps où j'ai eu l'occasion de réaliser des observations non participantes sans grille d'entretien aux côtés des professionnels exilés à Calais en 2015, puis en 2018 et 2019, seuls étaient notés les dates et tout ce que les sens de l'observateur pouvaient collecter sur l'instant (comportement, parole, gestes, réactions) avec cette difficulté de ne pas toujours saisir les échanges entre soignants exilés et patients exilés en raison de la barrière de la langue de l'observant. Des entretiens semi-directifs d'au moins quatre heures sont venus soutenir l'observation non participante. Les noms des soignants ont été changés pour cet article.

8 Cette méthodologie a toutefois de nombreuses limites, d'autant plus que le nombre de soignants exilés est restreint - ils sont quatre - ce qui ne permet pas de tirer des conclusions générales et cette recherche a donc un caractère très modeste mais en décortiquant la trajectoire professionnelle d'une soignante afghane en exil, depuis son départ d'Afghanistan jusqu'à son intégration à la PASS de Calais, cet article apporte des pistes de réflexions. À travers l'histoire de cette soignante, surgissent diverses thématiques telles la perte du statut professionnel, la difficile réintégration dans le monde médical, le vécu du recrutement dans le système de soins français, l'expérience à la PASS et le bénéfice retenu pour reconquérir une identité professionnelle.

9 Nous verrons donc dans un premier temps comment les soignants exilés comme les reclassés ont été une ressource pour l'hôpital qui a permis de prolonger une médecine des précaires souvent boudée. Cette catégorie de professionnels se rassemble et se ressemble car ils partagent l'épreuve de la déconnexion médicale qu'ils ont connue sur leur trajectoire professionnelle. L'exil pour les médecins afghans est une circulation contrainte, un crève-cœur qui les désaffilie de la société dont ils sont issus. Même s'ils peuvent paraître privilégiés par rapport aux autres exilés parce qu'ils sont soignants, il n'en demeure pas moins que leur chemin jusqu'à leur intégration à l'hôpital a été tourmenté et le reste jusqu'à leur titularisation. 
10 Dans un deuxième temps, nous verrons que les repères culturels perdus, du fait de l'éloignement avec leur pays, sont réanimés à la PASS où l'appartenance culturelle des médecins afghans devient un atout pour les soignants français; elle peut aussi constituer un désavantage dans la relation avec les soignés où des tensions peuvent éclater. Mais la compassion des soignants afghans pour des patients qui ont vécu comme eux l'exil permet de dépasser les dissensions.

\section{Soignants reclassés et professionnels exilés à la rescousse de l'hôpital}

11 Face à l'ampleur de l'urgence humanitaire en 2014 et pour augmenter les effectifs médicaux de la PASS, l'hôpital s'est orienté vers les soignants en situation de reclassement professionnel (harcèlement, maladie, épuisement...). Leur intégration à la PASS est une sorte de seconde chance après une trajectoire professionnelle fêlée. L'hôpital a aussi recouru à des praticiens ayant un diplôme hors Union Européenne (PADHUE). Ils sont quatre médecins - Dorreh, Dastan, Habibullah et Farhad - tous Afghans, parmi eux, Dorreh, la quarantaine, première femme médecin à la PASS et rare femme à exercer la médecine en Afghanistan. Elle a travaillé dans le milieu hospitalier à Kaboul en tant que gynécologue avec des conditions de travail précaires et compliquées à cause de la guerre (violence, kidnapping, attentat), du manque de moyens matériels, techniques et des risques de bombardements. Soignants reclassés et exilés ont donc prêté main forte à cette médecine des précaires encore déconsidérée.

\section{Soigner les exilés précaires : la double réticence}

12 L'adaptation à l'environnement social des patients est au cœur de la philosophie des PASS qui mettent en œuvre une médecine de type médico-sociale auprès de ceux que l'institution hospitalière nomme les "précaires ». Cette catégorie sociale est utilisée par l'hôpital et correspond aux patients dépourvus de couverture sociale et qui peuvent bénéficier, sous conditions de ressources, de soins gratuits. Cette expression, je la reprends dans mon travail au sens où Robert Castel l'entend lorsqu'il indique qu'ils sont en proie " à l'instabilité et à la montée des incertitudes » qui rejoint " l'incertitude migratoire " dont parle Smain Laacher au sujet des clandestins. Les exilés sont le résultat d'un délitement des cadres intégrateurs de la société. Dans sa forme ancestrale, l'hôpital accueille donc " ces gens de trop " (Castel, 1995) mais la création des centres hospitaliers universitaires ( $\mathrm{CHU}$ ) en 1958 a écarté quelque peu cette dimension sociale au profit des prouesses techniques et des spécialités. Dans ce contexte, des services comme la PASS sont devenus non attractifs pour des professionnels épris de "technique " ou réticents à soigner les précaires et donne lieu à penser que les professionnels de la PASS vivent en vase clos, en circulation fermée entre soignants compatissants à l'égard de la souffrance des précaires. Le personnel de la PASS de Calais est composé d'un personnel dit "volontaire»: il leur est proposé d'effectuer des observations dans le service avant de s'y engager. Pour beaucoup, c'est une découverte, même en travaillant à l'hôpital, ils n'avaient jamais entendu parler de la PASS. Car, à l'instar du secteur de la psychiatrie, ce ne sont pas des services hospitaliers prisés, cela ressort des entretiens réalisés auprès des professionnels de la PASS qui y ont travaillé sur différentes périodes (2006-2018). "On ne peut pas parler de jalousie vu les appels à

Revue francophone sur la santé et les territoires, Les circulations en santé : des produits, des savoirs, des personnes en mouvement 
candidature pour le poste et le nombre de candidats (rire moqueur). Les gens ne sont pas envieux ", constate une infirmière en entretien. D'ailleurs, un appel d'offres lancé par l'hôpital auprès des dentistes travaillant en libéral dans le Calaisis n'a reçu que deux réponses favorables, ce qui vient aussi conforter les propos de cette infirmière.

13 L'une des raisons est qu'à la PASS, les professionnels pratiquent la médecine générale qui dans les années 1980 s'est retrouvée à la marge au sein des hôpitaux et les soignants qui la pratiquaient «évolu[ai]ent en autarcie » (Baszanger, 1981). Plus récemment, Marc Bessin (2003) a montré que : "la médecine générale n'est pas ce qu'il y a de plus prestigieux à l'hôpital. Le système de domination dans l'institution hospitalière repose en effet implicitement sur une liaison forte entre spécialisation, sélection du recrutement et valorisation d'un service. "

14 C'est ce que j'ai pu remarquer auprès d'une infirmière des urgences en remplacement à la PASS de Dunkerque et qui vivait son affectation comme " une punition ». Selon elle, les soins n'étaient pas techniques comparés au service des urgences et elle s'ennuyait précisant: "Que faire à part coller des étiquettes sur les dossiers et faire de l'accompagnement?"

15 En second lieu, le désamour à l'égard de cette médecine s'ancre d'autant plus qu'elle s'adresse ici exclusivement aux précaires, qui plus est, sont en grande majorité des exilés et provoque une double réticence. En quelque sorte, s'occuper des exilés précaires pourrait compromettre le statut professionnel acquis. La constatation est la même en soin palliatif où Michel Castra (2003) a démontré que les professionnels qui y travaillent sont considérés par leurs collègues des autres services comme "déviants ». Comme ce service, la PASS " bénéficie d'un faible prestige » (Castra, 2003), les soignants seraient ceux qui occupent les " postes poubelle " (selon une infirmière de la PASS interrogée). D’ailleurs, les soignants de la PASS de Calais se sentent stigmatisés par les autres professionnels au sein de l'hôpital comme étant les " médecins des migrants "; pour autant, les premiers passent outre ces remarques négatives. Dans cette acception, s'occuper des exilés constituerait donc "un sale boulot » (Hughes, 2010) délégué au personnel de la PASS, un peu comme pour les services de soins palliatifs qui reçoivent des patients complexes considérés comme " difficiles " dans les autres services (Shepens, 2014). On retrouve ces formes de réticences à l'hôpital mais aussi en médecine libérale. Caroline De Pauw (2013) a par exemple montré dans son étude que les médecins de ville ont des difficultés à accueillir les précaires au point de penser qu'ils méritent une médecine spéciale: "un discours assumé par une partie du corps médical ferait de la précarité une "spécialité ", un exercice particulier à l'image de ce qui peut se faire pour l'alcoologie, la toxicomanie » Caroline De Pauw (2013).

Le rapport des professionnels à la précarité a fait l'objet de plusieurs travaux sociologiques qui ont montré que des soignants à l'hôpital peuvent exprimer du dégoût à l'égard de patients à la marge. Cette littérature relate les rejets à l'égard des toxicomanes aux urgences (Bessin, 2003), aux stratégies visant à décourager les patients précaires de rester dans le service (Peneff, 1992), aux soignants qui parlent des précaires comme de "mauvais malades" (Hassin, 1996). Ainsi pour l'hôpital les soignants exilés et reclassés constituent un réservoir pour faire perdurer une médecine marginale. 


\section{Trajectoires professionnelles fêlées : le point commun des soignants reclassés et exilés} (ORL) mais avec l'arrivée des talibans, elle doit se diriger vers la gynécologie parce que toucher des hommes, même dans un cadre médical, est interdit. Elle porte la burqa pour sortir. Grâce à des contacts hauts placés chez les talibans de certains de ses camarades qui, contrairement à elle sont Pachtounes, elle a pu continuer à faire ses études et terminer son stage dans un hôpital à Kaboul qui acceptait les femmes. Elle obtient sa spécialité et travaille pendant sept ans à la maternité. Elle décide de quitter le terrain pour rejoindre le ministère de la santé sous le gouvernement d'Hamid Karzhaï où elle travaille en tant que cadre sur la relation mères-enfants, elle repère les problèmes et propose des solutions, " il y avait beaucoup de défis! C'était un challenge!", mentionne-t-elle. Lors de son entretien, elle explique ne pas vouloir entrer dans trop de détails pour des raisons de sécurité et en tant qu'interlocutrice, je n'ai pas souhaité davantage franchir son intimité. de quitter son pays avec son mari et ses enfants. La guerre et plus précisément le sentiment de danger sont à l'origine de leur départ et constitue un "push factor " (facteur de répulsivité) indiscutable dans la prise de décision. L'Europe représente alors un espace-refuge où les exilés projettent de se reconstruire. On retrouve aussi chez les médecins africains sur lesquels Angèle Flora Mendy (2014) a travaillé, ce même sentiment d'insécurité qui explique cette « fuite des cerveaux ».

«J'ai quitté le Cameroun pour des raisons essentiellement politiques, parce que j'ai échappé à un assassinat politique dans un régime autoritaire. Je ne parle pas de l'environnement de travail qui était très dissuasif (...). Les autorités ont tout fait pour que le salaire d'un médecin soit largement inférieur au salaire d'un gendarme (...). Certains médecins sont mutés dans des zones isolées sans logement ni transport alors qu'ils ont besoin de conditions stables pour exercer la médecine. »

$\mathrm{Du}$ pays en guerre aux « lieux-frontières » (Cuttitta, 2015), la circulation des exilés s'inscrit dans un mouvement contraint. Sur la Côte d'Opale, à Calais et Grande-Synthe, la plupart d'entre eux sont considérés par l'Etat français comme « clandestins », ils sont "ceux qui ne sont pas les bienvenus» (Laacher, 2007). Nous les nommons dans cet article «les exilés». Ce terme renvoie au courant sociologique porté par Abdelmalek Sayad et poursuivi par Smain Laacher, l'exil est entendu ici comme un processus subi et le résultat d'une superposition de cassures depuis le départ du pays natal, il suppose la nostalgie, la mélancolie de sa terre, la séparation avec ses proches... Les corps des exilés sont "traversés ", un verbe polysémique qui possède d'abord un sens étymologique signifiant " parcourir un espace d'un bout à l'autr e » (Ortolang, CNRTL) et qui s'apparente à la double absence (Sayad, 1999), à l'arrachement culturel et affectif au pays (Laacher, 2005), à la mobilité contrainte, les exilés sont en situation de rupture radicale, de " perte du lieu dans le monde ", ils sont à " mi-chemin vers nulle part " (Bolzman, 2014). Ces corps traversent aussi des frontières au second sens de "se frayer un chemin à travers " (étymologie du XVI ${ }^{\mathrm{e}}$ siècle), c'est l'incertitude du voyage " comme une étape de plus d'un voyage qui n'en finit pas; où ils n'en finissent pas d'arriver. " (Laacher, 2005).

Revue francophone sur la santé et les territoires, Les circulations en santé : des produits, des savoirs, des personnes en mouvement 
Enfin, ces corps sont traversés par la frontière, " elles les percent de part en part " ( $\mathrm{X}^{\mathrm{e}}$ siècle) au sens symbolique, elle est un rempart contre lequel les exilés se heurtent, se blessent, meurent.

21 L'exil constitue un choc, une fêlure puisque " l'arrachement au pays » (Laacher, 2005) implique une situation de rupture radicale. Les soignants ont dû abandonner leur statut social pour se fondre parmi ceux qui se retrouvent sur les routes; ils deviennent comme les autres exilés, « des surnuméraires » (Castel, 1995). Les chemins de l'exil ont différents visages : ainsi, deux collègues de Dorreh se sont rendus en Europe par avion avec un visa et l'un d'eux s'est retrouvé dans des camps précaires en Normandie mais leur temps de transit n'a pas dépassé plusieurs semaines contrairement à d'autres exilés qui peuvent y rester des mois.

«Je ne pouvais pas aller en Angleterre qui est hors Schengen. Quand j'ai vu ce qu'il se passait à Calais ou à Cherbourg, j'ai vu que c'était très difficile. Je n'ai pas essayé, j'ai dit : « Non, je ne peux pas ». C'était très dangereux. J'ai entendu qu'il y avait eu trois morts en deux semaines, je n'ai pas osé tenter J'ai donc choisi de rester là.» Habibullah, 41 ans, collègue de Dorreh.

22 A l'inverse, Dorreh et sa famille n'ont pas pris l'avion. Là encore, elle ne souhaite pas s'étendre avec précision mais en filigrane, je comprends qu'elle a traversé beaucoup de pays, " c'est un voyage clandestin, pas volontaire ", passant illégalement les frontières par camions et a connu la précarité. " J'ai traversé beaucoup de pays, je suis passée par le même chemin que mes patients ", précise-t-elle. Lors de l'entretien, Dorreh a indiqué que parler de son parcours migratoire était quelque chose " de trop douloureux pour être répété ", que cela ne lui a pas " laissé un bon souvenir ", qu'elle préfère " oublier ». Dorreh perd son statut professionnel et le contact avec la médecine pendant l'exil. En entretien, cette rupture avec le monde médical est pour elle source de stress car elle s'inquiète de perdre des années, de ne plus pouvoir rattraper sa carrière et exprime son manque d'un métier qu'elle ne peut plus exercer.

Cette fêlure, on la retrouve chez les soignants reclassés. Elle a pris la forme d'un sentiment de mise à l'écart chez Françoise, 54 ans, infirmière reclassée à la PASS. Elle a travaillé au bloc opératoire comme référente hygiène, elle s'est retrouvé en burn out avec un arrêt de travail de six mois qui la déconnecte du monde médical hospitalier.

Soignants exilés et reclassés saisissent alors la PASS comme une opportunité pour réintégrer un monde médical qu'ils ont dû mettre entre parenthèse.

\section{La reconnexion avec le monde médical}

Les épreuves pour intégrer l'hôpital se juxtaposent à celles engendrées par l'exil. Le système de soin français est très réglementé, plus encore pour les "praticiens à diplôme étranger hors Union Européenne » (PADHUE).

\section{« Être soignant » : un marqueur en surbrillance}

Avant de pouvoir exercer, les PADHUE doivent régulariser leur situation administrative et obtenir le statut de réfugié. Dorreh indique que l'obtention de son statut de réfugié s'est effectuée rapidement. Elle a pour autant dû faire face à deux défis : celui d'apprendre la langue française et de retrouver un emploi. "J'ai commencé une vie plus difficile que celle dans mon pays. " Comme les autres soignants afghans, il leur a fallu maîtriser le français et c'est souvent auprès d'associations qu'ils l'apprennent. Ce 
que regrette d'ailleurs Dorreh qui indique qu'elle s'est retrouvée mélangée avec des personnes analphabètes dans des cours où la différence de niveaux était flagrante, elle exprime sa frustration de ne pas avoir pu bénéficier « d'un niveau académique, le niveau était trop bas, j'ai perdu beaucoup d'années pour apprendre la langue ".

Elle ajoute: « Toujours on me demandait de savoir: "Combien j'achète de carottes? " ou «Combien je vends ceci? » Mais je leur ai dit : «Je ne suis pas là pour faire mon marché! Je suis une femme médecin, j'ai besoin d'un bon niveau de langue. "

Elle a donc utilisé ses économies pour financer une formation accélérée dans une université française, tout en suivant des cours de français dans plusieurs facultés. " J'étais toujours avec mes livres devant la télévision, je m'occupais moins de mes enfants. J'avais peur de perdre du temps pour ma carrière. "

29 Pour obtenir le statut de praticien exigé par la France, cela relève du parcours du combattant. Les PADHUE peuvent reprendre des études à l'université. Farhad, un collègue afghan de Dorreh, en retient une expérience négative :

«On m'a dit qu'il fallait que je me réinscrive en première année et que si je réussissais le concours, au lieu d'aller en deuxième année comme les autres étudiants, j'irai directement en dernière année. C'était impossible. Et puis, les étudiants de première année ont 17 ou 18 ans, moi j'ai eu mon bac il y a douze ans. Alors je me suis dit que j'irai valider mes diplômes en Angleterre. »

L'autre solution est de passer la procédure d'autorisation d'exercer (PAE) qui est accordée par le ministère de la santé, démarche qu'ont choisi trois soignants exilés de la PASS. Cette PAE exige des prérequis. Ils doivent prouver qu'ils ont bien exercé en Afghanistan, passer un examen écrit de vérification des connaissances pratiques et fondamentales où il est stipulé qu'une bonne connaissance du français est indispensable. Le niveau B2 de français d'expression à l'oral est obligatoire. A la suite de cette validation, ils enchaînent avec une période probatoire de trois ans sous la responsabilité d'un chef de service agréé dans la formation des internes. Les dossiers sont examinés par la commission PAE : 941 dossiers ont été examinés en 2016 et $72 \%$ ont reçu un avis favorable (Bouet, 2017). Il faut ensuite être inscrit au tableau départemental de l'ordre des médecins. On comprend que cela nécessite d'avoir de grandes ressources en soi pour ne pas décrocher, les soignants exilés sont mis à l'épreuve.

31 Le parcours de Dorreh pour trouver du travail a été laborieux. Elle explique avoir démarché plusieurs hôpitaux parisiens pour trouver un emploi mais a essuyé les refus. $\mathrm{Au}$ Pôle emploi, elle indique qu'on lui proposait toujours des postes de femmes de ménage. Il a fallu qu'elle attende 2015, quatre ans après son arrivée en France, pour renouer avec le médical. C'était donc à Calais pendant la «new jungle » et parce qu'à l'époque, son mari avait déjà été embauché comme médecin associé lauréat PAE pour la PASS-Ferry. Le médecin-coordinateur - qui est à la fois urgentiste et gériatre, salarié depuis les années 1990 à l'hôpital de Calais - lui a demandé si Dorreh pouvait intervenir sur une épidémie de varicelle. Elle a été recrutée sous statut de faisant fonction d'interne (FFI) et détaille son choix :

"J'ai passé des entretiens avec la direction et le chef de la PASS, on m'a expliqué les conditions, les procédures de prise en charge des patients finalement j'ai accepté même si cela me semblait pas très intéressant pour moi comme j'ai une expérience de huit ans de gynécologie. Mais maintenant je suis trop contente parce que je sens que je suis très utile ; j'ai trouvé que ce pan de la médecine était pas mal. » 

repère et qui se démarque ; de même, ici, il semble que la profession médicale agit ainsi sur les bénévoles dont l'attention est alors plus accrue. "Être soignant » permet ainsi aux exilés de se démarquer, de devenir singulier parmi les surnuméraires. Les exilés soignants semblent durant leurs parcours «avoir plus de chance » si on peut dire que les autres. Mais cela ne les rend pas indépendants pour autant, au contraire : dans une société qui prône la capacité « à se tenir face au monde » et " avoir la propriété de son soi »(Martuccelli, 2002), ces médecins afghans sont dans une situation de dépendance visà-vis de potentielles bonnes âmes qui leur tendraient la main.

\section{La reconquête du statut professionnel}

Les soignants afghans forment une minorité parmi les médecins étrangers puisque leurs origines n'apparaissent pas parmi les dix principaux pays des médecins nés en Europe ou en dehors de la communauté européenne et exerçant en France (Le BretonLerouvillois, 2014). Leur nationalité est donc étonnante dans ce panel de profils de soignants et en effet, la plupart des médecins hors communauté européenne proviennent surtout de pays anciennement colonisés ou placés sous protectorat 
français (Algérie (25\%), Maroc (11,5\%), Liban $(4,4 \%))$. Ces PADHUE atypiques sont ancrés dans un processus de reconstruction professionnelle et de reconquête de leur statut. Dorreh a échoué à l'examen de PAE en France et a été recrutée en 2015 sous un statut spécifique qui lui a permis d'exercer à la PASS même sans avoir été lauréate PAE. La circulation des médecins étrangers vers une ascension professionnelle est rendue possible grâce à l'attention portée au sein de l'hôpital par des professionnels de l'hôpital ou grâce aux "bons plans" que vont s'échanger les soignants exilés expérimentés avec les novices et aussi aux assouplissements et marges de manœuvre de la direction. Un cadre indique d'ailleurs que l'institution hospitalière agit aussi comme un soutien même si son action a des limites :

« La direction de l'hôpital et la direction aux affaires médicales ont défendu la cause de deux médecins auprès de la préfecture qui avaient des titres de séjour reconduits tous les mois ou tous les trimestres et sur lesquels pour avoir un vrai contrat de travail, il nous fallait une vraie régularisation de la préfecture.»

Le cas mentionné a été voué à l'échec puisque " l'hôpital n'a pas de prise sur ces questions » mais la démarche institutionnelle est là et peut s'avérer plus fructueuse au sein de ses murs. On voit bien que la souplesse administrative a été bénéfique à Dorreh, recalée au concours PAE et à qui a été proposé de pratiquer la médecine générale sous statut de faisant fonction d'interne (FFI). Dorreh peut, peu à peu, raccommoder la fêlure. Ce soutien de l'hôpital, Françoise l'a pratiqué lorsqu'on lui a proposé à la fin de son burn out de travailler à la PASS : "C'était un bâtiment à l'écart, c'était bien comme je ne voulais plus retourner dans le bâtiment principal. "

39 Pour les soignants afghans qui exerçaient dans des services spécialisés en Afghanistan, la PASS n'est pas leur vœu de prédilection néanmoins elle assure un retour vers la médecine hospitalière et les place dans une dynamique de reconquête de leur statut professionnel. Les soignants étrangers qui tiennent un « rôle d'ajustement » (Cottereau, 2018), utilisent cette caractéristique pour trouver une place. Les soignants exilés espèrent que la PASS puisse constituer un tremplin, une première étape qui les porte vers leur spécialité et perspectives d'emplois, de carrières à long terme avec un vrai statut professionnel.

L'expérience à la PASS est aussi une première approche pour se familiariser avec les pratiques professionnelles liées au système de soin français. Les soignants afghans évoquent surtout l'apprentissage des protocoles standardisés. Il arrive que Dorreh vienne voir le travail des infirmiers en salle de soin et demande à les aider pour apprendre des protocoles.

«Je lui montrais comment faire un pansement, quel matériel utiliser, les produits par exemple les médicaments à quoi ça correspondait, les antiseptiques, les bandages. » (Une infirmière)

41 Les médecins afghans ont fait des études dans des universités créées entre autres par la France ou dont le contenu des cours est influencé par l'Occident (Jonquet, 2005), que le modèle sanitaire du pays fonctionne beaucoup grâce aux aides des ONG. Cependant en France, ils doivent s'adapter à un modèle sanitaire qu'ils estiment de manière positive "plus strict» sur les normes et les réglementations (Azria, 2013 ; Belorgey, 2010). Le mari de Dorreh le résume bien :

«La médecine ici est plus standardisée, les traitements et aussi les protocoles, les bilans sanguins pour le patient, sur cela oui j'ai appris. L'hôpital a des protocoles par exemple pour le traitement de [la] gale, c'est spécifique et aussi pour la douleur et les infections, on ne peut pas prescrire un antibiotique comme cela, il y a un 
protocole. En Afghanistan, il n'y a pas beaucoup de protocoles. C'est plus de liberté mais ce n'est pas bien en termes de santé publique, car très vite le médecin va prescrire un antibiotique et c'est comme ça que cela crée des résistances. » d'antibiotiques, il leur a alors conseillé de diminuer cet usage systématique. Pour apprendre ces nouvelles normes, les médecins afghans s'appuient sur le corps infirmier présent quotidiennement. A la PASS, l'organisation est surtout collaborative plus que hiérarchique; les infirmiers deviennent de véritables référents pour ces soignants exilés. Habibullah, le collègue de Dorreh, en témoigne :

«Si j'ai une difficulté, si je veux savoir quelque chose j'appelle le médecincoordinateur, c'est lui le chef de la PASS. On est une équipe, si je ne sais pas des choses sur la pharmacie ou si quelqu'un a besoin de voir un spécialiste, je demande aux infirmiers comment on peut gérer cela, eux m'indiquent par exemple tel addictologue, ce que l'on fait pour les patients suicidaires, je vois avec les infirmiers qui ont plus d'expérience et ça fait des années qu'ils travaillent ici, ils savent comment organiser ça. »

En plus de l'apprentissage, l'expérience à la PASS compte dans la période probatoire et permet aux soignants exilés de valider leur quota d'années qualifiantes. Le mari de Dorreh, Dastan espère qu'après ses trois années de probation, il sera titularisé. Après son expérience à la PASS, Dorreh a été inscrite en 2018 dans un plan de formation pendant un an qui lui permet d'exercer dans un service de gynécologie, " car ça [lui] manquait [sa] carrière de gynéco ». Elle travaille la semaine à Soissons situé à $2 \mathrm{~h} 30$ de Calais, dort dans une petite chambre d'interne et retourne en train chaque week-end pour voir sa famille à Calais. Elle compte retenter l'examen de PAE pour pouvoir exercer comme gynécologue à Calais. "On espère une fois sa formation terminée, qu'elle récupèrera un poste de gynécologue à l'hôpital, aussi à la PASS et au centre gratuit d'information et de dépistage et de diagnostic (CeGIDD) où nous avons des consultations pour des maladies sexuelles ", souligne le médecin coordinateur de la PASS. Dorreh possède comme les Brésiliens étudiés par Françoise Chamozzi - un niveau de qualification élevée qui agit comme "un outil de progression» d'un temps de précarité vécu à l'arrivée en France vers semble-t-il une " success story » (Chamozzi, 2009). Pour autant, l'expérience en France n'a pas été ressentie de la même manière par son collègue Farhad. Les procédures drastiques et les questions éthiques que posent les recrutements (Drexter, 2008) et la différence de statuts entre soignants français et étrangers lui ont laissé un sentiment de stigmatisation, d'infériorisation, de médecin de seconde zone. Il a préféré partir en Angleterre où il est plus aisé d'atteindre des « carrières réussies » (Mendy, 2014). La trajectoire professionnelle de Farhad, parti en Angleterre, incarne aussi les failles du système de soin français. Il décrit :

« À la différence de la France, en Angleterre, j'ai le même statut que quelqu'un qui a fait ses études à l'université d'Oxford, il n'y a pas de différences, j'ai le même salaire. Pour entrer dans une spécialité, le recrutement est national, une fois que vous avez vos équivalences, on ne vous demande pas d'où vous venez, qui vous êtes, votre passé, il n'y a pas de questions personnelles du tout, c'est le même entretien pour tous. Même si vous êtes français par le passeport, on va vous rappeler que vous n'êtes pas de ce pays où que vous étiez réfugié avant. "

Le recours à ces deux catégories de soignants - les reclassés et les exilés - a donc été une aubaine pour l'institution hospitalière. Cette circulation ouverte a permis de constituer un agrégat original composé d'une diversité d'identités professionnelles.

Revue francophone sur la santé et les territoires, Les circulations en santé : des produits, des savoirs, des personnes en mouvement 
Nous verrons dans cette deuxième partie comment la culture des soignants afghans a été mobilisée à la PASS.

\section{Transmission des savoirs : les soignants exilés, des passeurs culturels}

La PASS, durant le temps de la «new jungle » (2014-2016) a réuni des médecins français issus de la médecine libérale qui partagent une sensibilité pour l'humanitaire, sont en majorité engagés dans des associations, avec des paramédicaux hospitaliers (en situation de reclassement ou pas) généralement non engagés dans l'humanitaire même s'ils ont une ouverture à l'autre, des médecins hospitaliers (français et afghans), des représentants de l'établissement de prévention aux urgences sanitaires (EPRUS) présents pour soutenir l'hôpital dans la création de la PASS-Ferry. Cet agrégat d'expériences professionnelles singulières amène les soignants à partager leur savoir. Pour illustrer ce constat, un infirmier a appris à ses collègues de la PASS le protocole des grands brûlés qu'il utilisait lorsqu'il travaillait dans le service de traumatologie.

La nature des interactions entre les professionnels est extrêmement variable puisqu'elle dépend de la configuration des lieux (bâtiment unique ou modulaires séparés) et du rythme de travail. Ainsi au début de la PASS-Ferry (environ cent consultations par jour pour deux médecins), les professionnels mangeaient en cinq minutes et reprenaient leur travail immédiatement; un rythme qu'ils ont fini par ne plus supporter. Ils se sont donc obligés à faire de vraies pauses repas ce qui leur a permis de se connaître, d'échanger et de se rapprocher.

\section{La différence culturelle, entrave à la circulation des soins entre soignants français et patients exilés}

Les soignants qui travaillent à la PASS ont pour la plupart une sensibilité pour la dimension humanitaire : certains ont effectué des missions en Afrique, ont créé leur association humanitaire en France, se sont portés bénévoles auprès de Médecins du Monde ou Médecins sans frontières qui a notamment œuvré auprès des exilés sur le littoral avant 2006, pour d'autres l'humanitaire était un rêve.

Durant leurs études, les médecins apprennent la médecine tropicale tout au plus ce qui oblige la plupart des professionnels à se former sur le terrain pour soigner les exilés comme les paramédicaux qui indiquent avoir «appris sur le tas». On a par exemple une infirmière qui a réalisé un court mémoire sur les différentes communautés qu'elle recevait à la PASS et avait noté leurs caractéristiques, elle a passé un diplôme en psychiatrie transculturelle. Elle indique :

«Moi, l'Afghanistan, bah j'en connaissais pas grand-chose et puis j'ai découvert qu'il y avait des Hazaras, des Pachtounes et des Tadjiks, qu'ils ne parlaient pas la même langue, que les Pachtounes, ils avaient le dessus sur les Hazaras, je l'ai constaté de fait à Calais et mes études en psychiatrie transculturelle m'ont permis de comprendre ce que j'observais à Calais. "

Ces soignants sont curieux d'apprendre et sont en quête de notions sur la culture de leurs patients en provenance de l'Afrique et du Moyen-Orient : certains se documentent 
en lisant des ouvrages sur la traversée des exilés, d'autres en regardant des documentaires, d'autres encore en suivant l'actualité au Moyen-Orient.

Pour autant, malgré ces démarches «d'aller-vers », ces qualités ne suffisent pas pour dépasser les freins en jeu dans la différence culturelle et parvenir à réaliser une médecine approfondie. D'abord, il existe des situations où les maladies (paludisme), les représentations de la maladie (faille dans l'organisme en France, mauvais sort ou punition en Afrique) ne sont pas les mêmes (Laplantine, 1986). Ainsi, des soignants se sont étonnés que leurs patients réclament des injections (qui se pratiquent beaucoup en Iran par exemple) plutôt que des traitements par voie orale. Un médecin était déconcerté de voir qu'une de ses patientes n'avait pas eu ses règles pendant trois mois suite à une injection contraceptive -très répandue en Afrique - administrée en Libye et qui constitue une solution de dernier recours pour les personnes précaires exilées (Bretin, 2004). Les soignants exilés témoignent aussi des différences culturelles dans le champ médical. Dorreh raconte: "Sur les problèmes sexuels, ici en France, les patients français sont très ouverts, ils demandent beaucoup de choses alors que chez nous les gens hommes et femmes - n'aiment pas les réponses directes, il faut choisir un chemin détourné pour pouvoir parler à la personne. "

51 Lorsqu'elle s'occupait des femmes enceintes afghanes, la plupart arrivait à l'hôpital avec des complications voire des urgences vitales ce qui est rarement le cas en France. Elle explique aussi avoir été surprise que des césariennes soient systématiquement réalisées lorsque la présentation du bébé se fait par le siège précisant que dans son pays les femmes accouchent quand même par voie naturelle. Elle ajoute aussi que les échographies sont réalisées seulement en cas d'urgence, or en France, elle a dû apprendre à conseiller plus fréquemment ces nouvelles technologies.

52 Plus évidente, la barrière de la langue est un frein majeur souligné par les professionnels eux-mêmes et qui produit une réelle frustration. En effet, malgré la proximité avec l'Angleterre, les soignants ont quelques notions d'anglais et ils s'appuient surtout sur l'interprète afghan. Mais cet intermède peut à la fois être un soutien (" le traducteur soulage et permet un bon interrogatoire ", précise un médecin) et une contrariété puisque cette relation soignant-soigné à deux doit être alors repensée à trois. L'interprétariat dans le soin ne fait pas l'unanimité. La question de la précision de la traduction est posée, celle de la disponibilité de l'interprète aussi.

«Exprimer le ressenti de quelqu'un sur le plan psychologique reste difficile quand il

$\mathrm{y}$ a un interprète parce que les mots qu'elle va utiliser, ce sont des mots qu'elle va peser et soupeser mais l'interprète ne va pas forcément transmettre de façon pieuse. » Un médecin libéral

L'interprète assure plusieurs tâches à la PASS : traductions des consultations médicales, psychologiques, l'accompagnement des patients vers les services spécialisés. Il arrive souvent que des médecins se retrouvent seuls face à leurs patients sans pouvoir leur parler. Alors ils bricolent en usant du mime.

« On fait le singe !, indique en plaisantant une dentiste. Si on veut qu'il garde la bouche ouverte, on montre en haut, pour fermer, en bas, on fait beaucoup de gestes. Pour les enfants, c'est plus difficile parce qu'ils pleurent et sont plus angoissés alors on parle avec une voix plus posée, on chantonne. »

54 Des stratégies de communication ont été pensées pour surmonter les freins à la communication comme le collage d'autocollants avec un soleil (le midi) et une lune (le soir) sur les boîtes de traitements afin que les exilés puissent repérer les temps de prise des médicaments. L'hôpital a aussi fait traduire en plusieurs langues (pachtou, ourdou, 
tigrinya, etc.) les plans de la PASS et développé des pictogrammes pour indiquer qu'un psychologue est présent, que des tests pour les maladies sexuellement transmissibles sont possibles. Mais la communication reste limitée malgré les moyens mis en œuvre, ce qui renforce l'asymétrie de la relation soignant-soigné.

Le respect de la pudeur des patients peut aussi limiter l'échange médical. En effet, des soignants indiquent ne pas forcément insister pour comprendre ce qui ne va pas chez leurs patients pour éviter de les braquer parce que l'hôpital est une institution étatique qui génère une méfiance chez des patients qui craignent d'être dénoncés à la police. De plus, les soignants ne veulent pas non plus raviver des traumas vécus par les patients. Tandis que les professionnels de santé hospitaliers, qui se savent considérés comme des représentants de l'Etat, tentent aussi de mettre en place une relation de confiance avec leurs patients et le fait de ne pas insister témoigne aussi de cette volonté de respecter les patients dans leur intimité.

« Il y a des patients qui ne veulent pas parler de leurs problèmes. Les gens ne veulent pas rentrer dans leur intime, ils l'expliquent clairement. (...) Il y a aussi la peur, des gens qui ne veulent pas communiquer sur leur passé par peur que ce soit divulgué (...) Il y a aussi des gens qui ne veulent pas entrer dans leur vie parce qu'il y a de la souffrance sur leur parcours, ils ne veulent pas rajouter une souffrance sur une souffrance donc ces douleurs, ils préfèrent les enfouir ", témoigne un médecin hospitalier.

Le respect de la pudeur entre aussi en contradiction avec un système sanitaire qui encourage les patients à se dire, à raconter leur biographie (Astier \& Duvoux, 2006) où le trauma psychologique est devenu une arme pour l'obtention de droits et la psyché un espace de revendication (Fassin \& Rechtman, 2007), ce modèle « d'injonction à se rendre visible » (Aubert \& Haroche, 2011). Des professionnels précisent que leurs patients viennent surtout pour obtenir un soin brut pour rétablir leur corps plus que leur état moral de sorte qu'ils puissent continuer leur trajet.

\section{Les interactions interculturelles pour dépasser les frontières du soin}

57 L'arrivée des médecins afghans a été perçue par les soignants français comme un atout. La PASS est un lieu presque familial où se joue l'entraide et la solidarité au sein de l'équipe soignante. Le local est constitué d'un couloir central qui relie les différents bureaux, la topographie des lieux favorise les échanges. A la PASS, nous sommes au cœur de la sociologie d'Anselm Strauss, l'endroit favorise le croisement de trajectoires professionnelles distinctes qui produisent une co-construction singulière, « un segment » (in Baszanger, 1992).

58 Ces médecins afghans manient au minimum cinq langues, les dialectes communautaires (ourdou, pachtou, dari...) et les langues étrangères (anglais, arabe, etc.) Des soignants français s'appuient sur les médecins étrangers pour que ceux-ci traduisent leurs propos aux patients. "On avait la chance d'avoir des interprètes qui parlaient quelques langues et les médecins aussi. " (Une infirmière) Le médecin coordinateur de la PASS reconnaît que les soignants étrangers permettent de communiquer avec la majorité des patients et soulagent aussi l'organisation de travail ; l'interprète peut ainsi se concentrer sur d'autres tâches et soutenir d'autres professionnels de santé (psychologue, infirmier à la pharmacie, etc.) Les soignants exilés sont conscients eux-mêmes de leur apport. Cette aide à la traduction vient combler les carences de la société d'accueil en matière d'interprétariat ce qui évite 
aussi que l'échange se cantonne à une "relation vétérinaire " (Tabouri, 2009). Le mari de Dorreh souligne qu'il " connaît déjà la culture des gens qui sont là-bas. (...) C'était intéressant pour l'hôpital et pour moi aussi ». Dorreh qui comprend la culture des Irakiens ne s'est pas étonnée de voir un patient parler un mélange de kurde et de perse, " je lui ai dit que je le comprends et ça l'a mis en confiance ". Farhad explique que lorsqu'il était interprète, il a vu des soignants donner des traitements qui n'étaient pas les bons à cause de la barrière de la langue, " moi je peux expliquer les problèmes, c'est très important de savoir parler leur langue, je pouvais vraiment aider ces personnes ». Les soignants afghans peuvent aussi détailler le fonctionnement du système de soin français à leurs patients. Le mari de Dorreh indique qu'il a reçu à la PASS beaucoup de patients afghans originaires de villages dont les accès aux hôpitaux ont été rendus impossibles à cause de la guerre ; ainsi beaucoup ont eu recours à la médecine traditionnelle comme l'usage de tamaris , cette plante médicinale utilisée par les paysans afghans (Younos et al, 2005).

59 On voit aussi qu'il n'est pas toujours aisé pour les soignants français de voir comment s'y prendre avec des patients étrangers. Sur les rapports hommes-femmes par exemple. Pour une infirmière, son expérience à la PASS a été l'occasion de travailler pour la première fois avec des soignants étrangers. " Ça m'a permis de m'imbiber un peu des coutumes, sur la relation homme-femme déjà, de savoir un peu comment je devais me positionner par rapport au patient ", commente-t-elle en précisant qu'elle a beaucoup observé les soignants exilés pour adopter la bonne attitude à l'égard de ses patients.

60 Sur la prise en charge psychiatrique aussi. Farhad raconte qu'il a expliqué aux soignants français la manière dont il fallait dire à un patient qu'il est atteint de maladie psychiatrique précisant qu'en Afghanistan reconnaître cela c'est le réduire à la figure du fou et donc de celui qui ne bénéficie pas d'aide et qui est isolé. Il précise qu'il ne faut pas employer des termes médicaux tels que " dépression " mais plutôt mobiliser le registre du sensible et des émotions telle que la tristesse. Ainsi les soignants afghans transmettent certains codes aux soignants français, ce qui permet à ces derniers de se rapprocher de leurs patients.

61 Sur l'histoire des patients enfin. Les soignants exilés saisissent les subtilités émises par les patients ce qui permet de reconstituer le contexte. Un médecin français hospitalier raconte: "J'ai pu comprendre que la grand-mère que j'avais en face de moi avait perdu son mari, son fils et son neveu. J'ai pris conscience de l'ampleur de sa souffrance. J'étais moins déphasé. "

Ainsi, en apportant aux soignants français un éclairage culturel, les soignants réduisent l'écart qui existe entre soignants français et patients exilés, ceux-ci devenant de moins en moins étrangers. Ils peuvent aussi aller plus loin dans la relation avec les patients notamment en recevant leurs confidences sur les violences policières par exemple. Les soignants exilés mobilisés pour combler une carence organisationnelle renversent le stigmate. À travers ce rôle informel qu'on leur donne et qu'ils se donnent de "passeurs culturels ", ils deviennent un atout et présentent des qualités au sein de cette prise en charge des exilés précaires.

63 Mais cette appartenance culturelle des soignants exilés qui constitue dans la relation entre professionnels, peut aussi se retourner contre eux au sein de leur relation avec les soignés exilés. 


\section{L'appartenance communautaire, frontière et porosité dans la circulation des échanges entre soignants et soignés exilés}

afghanes en France. Les observations montrent qu'elles existent dans la relation soignant-soigné. Dans le même temps, nous verrons que la condition sociale des patients touche davantage les médecins afghans qui ont partagé le même vécu avec "plus ou moins de chance » dans l'exil. Le maintien de la distance professionnelle est alors un vrai défi. Mais cette épreuve commune peut aussi amener les médecins étrangers à s'investir davantage.

\section{Tensions et « bonne figure »}

Être afghan ne présente pas que des avantages et peut aussi poser des difficultés dans la prise en charge sanitaire auprès des exilés. Cela peut créer des réticences de la part des patients. Même si elles restent rares, elles existent. En 2018 par exemple, un patient pachtoune a refusé d'être soigné par un médecin afghan qui n'appartient pas à la même communauté que lui. En 2005, j'ai pu observer qu'un patient africain, probablement originaire du Soudan, a marqué un pas de recul en voyant dans la salle de consultation un médecin afghan. Il a alors demandé aux infirmières s'il pouvait passer avec un autre médecin. A l'arrivée du professionnel afghan qui s'est étonné de ne pas recevoir ce patient, les infirmières ont formulé un faux prétexte afin de ne pas le blesser.

Entre soignants, je n'ai pas eu l'occasion d'assister à des tensions lors de mes observations. En entretien, Dorreh explique que cela arrive parfois qu'il y ait des tensions mais rien de comparable à ce qui pouvait se passer en Afghanistan à l'hôpital où elle indique que certains professionnels décidaient de ne plus soigner les patients qui n'appartenaient pas à leur communauté. Un médecin tajik a par ailleurs indiqué qu'à Calais, le collègue avec qui il s'entendait le mieux était pachtoune.

« En Afghanistan, il existe un problème ethnique mais ici je ne pense pas parce que d'abord vous êtes étranger ici et c'est très important pour l'hôpital et l'équipe cette égalité entre tous, la discrimination n'est pas éthique, beaucoup de gens n'aiment pas ça.»

Dorreh raconte à propos de son expérience passée en Afghanistan :

«On a travaillé comme une équipe, il n'y a aucun signe, de mauvais signes par rapport à la religion. La dernière année en Afghanistan, le conflit politique a séparé les gens, créé des conflits entre les gens, j'ai constaté beaucoup de médecins qui n'avaient pas un comportement très professionnel, ils privilégiaient des patients qui parlaient la même langue ou provenaient de la même région. Moi, je n'ai jamais pensé que celui qui est devant moi est blanc, hindou, juif, j'ai jamais eu cette idée dans ma tête dans toute ma vie. Lorsque j'étais devant un patient, j'étais comme un médecin, ça a créé un grand conflit entre moi et les collègues. »

L'univers hospitalier et ses règles éthiques viendraient-elles ainsi gommer ou contrecarrer les différences et tensions qui ont pu exister en Afghanistan? Il est vrai que les soignants ont à cœur ce principe de soigner sans distinction qui est aussi martelé par l'hôpital français. Mais il ne faut pas oublier qu'en France, ces soignants exilés sont en période de reconstruction professionnelle qui, on l'a vu est difficile à acquérir, si bien qu'on peut formuler aussi l'hypothèse que cette mise en situation test 
les oblige aussi implicitement à faire profil bas et faire « bonne figure » afin d'éviter les faux pas.

\section{Le risque de «l'effet miroir»}

Soignants et patients ont un vécu commun - l'exil - cette réciprocité pose la question de l'implication émotionnelle de ces soignants exilés. Une des difficultés pour eux réside dans le risque "d'effet-miroir ", c'est-à-dire d'identification à l'autre, ce qui peut entraîner un risque de dérive vers un sentiment de rapprochement, d'attachement voire d'appartenance alors que le métier de soignant exige aussi une distance émotionnelle avec les patients. Interrogé sur cette ressemblance avec les patients, un médecin reconnaît avoir dépassé le cadre professionnel. A plusieurs reprises, il a donné de l'argent à des patients pour les dépanner malgré les mises en garde de l'équipe. Il précise avoir été touché par la détresse de son patient : " Je sentais qu'il était vraiment dans le besoin, c'est humain."

Deux autres médecins expliquent qu'ils essaient au mieux de garder cette distance professionnelle :

«Pour tous, c'est une histoire tragique donc on ne peut pas dire qu'on est plus touché par un Africain qu'un Afghan ou un Iranien, leur histoire n'est pas différente, elle est tragique. » Le mari de Dorreh.

71 Pour autant, par rapport à un patient français, ils indiquent qu'il se joue quelque chose de fort au niveau émotionnel avec des patients exilés et cette impression est bien exprimée dans ces propos :

«Émotionnellement, j'étais plus proche des migrants car je comprends bien leur problème que j'ai vécu mais au niveau professionnel, je ne fais pas de différence. Par rapport aux Français, les migrants ne connaissent pas beaucoup de choses alors je leur donne des conseils même si je ne suis pas obligée de le faire mais je leur donne des éléments importants comme sur comment se soigner ou se protéger. Mon vécu m'aide à les soigner parce que je comprends bien leur problème.» Dorreh.

72 En entretien, Dorreh qui indique soigner en donnant tous ses sentiments, fait un lapsus en employant le terme "enfants " pour parler de ses patients. A priori, les soignants exilés semblent donc être plus exposés émotionnellement. Ce constat renvoie à la notion de bonne distance dont parle Catherine Mercadier (2008) qui énumère ce qui, dans un service, participe à la mise à distance avec les patients (les moyens techniques, les gants, etc.) ou encore l'exemple que cite Nicolas Belorgey (2010) d'une soignante qui accueille les patients à la chaîne pour éviter de s'y attacher. Or, les moyens organisationnels de mise à distance sont présents a minima dans la médecine des précaires qui est une médecine générale. La technique qui crée un éloignement des soignants avec le corps de leurs patients n'est pas développée à la PASS où les moyens ne sont pas sophistiqués et où le toucher des corps produit un contact direct (Tisserand \& Rodriguez, in Cabral et al., 2019). De même la ritualisation des soins et des protocoles joue en faveur de cette mise à distance mais est à la PASS faiblement mise en œuvre : ce sont surtout les infirmiers qui réalisent des protocoles de soin si bien que les médecins semblent potentiellement plus exposés émotionnellement et ceux qui sont exilés le sont davantage encore que les médecins français. Catherine Mercadier rappelle que " la bonne distance, spécifique de la relation soignant-soigné, varie en effet selon le contexte ». Le vécu des individus et leur culture sont des paramètres qui modulent le curseur de la "bonne distance". Sans oublier le terme de proxémie qui recouvre cette idée que la 
distance entre deux individus est un construit culturel et subjectif (Hall, 2014). La culture des soignants et l'expérience d'exil partagée avec les patients sont deux dimensions susceptibles d'affaiblir l'armure émotionnelle nécessaire à la pratique du métier.

73 Paradoxalement, on constate aussi que la détresse des patients peut constituer un moteur qui permet aux professionnels exilés de tenir sur la durée en nourrissant l'acte de soin de sens. Les entretiens révèlent d'ailleurs qu'ils ont été surpris du traitement réservé aux exilés en France. Dorreh confie en entretien qu'elle a dû changer l'image qu'elle avait de la condition des exilés ici : "Je ne savais pas que c'était comme ça, ce n'était pas agréable, c'était très gênant » en parlant des conditions de vie réservée aux exilés en France. Elle ajoute : " J'ai découvert que les gens qui vivent dans la "jungle", c'est vraiment une vie dans la «jungle» comme au Moyen Âge ». Les soignants exilés sont donc à la fois acteurs et spectateurs des conditions de vie réservées aux exilés en France.

74 Comme pour les soignants reclassés, le degré de vulnérabilité des patients suscite leur empathie; ils semblent s'impliquer davantage. Dorreh précise: "J'ai essayé de comprendre pourquoi le patient est agressif", de "trouver un chemin pour être plus près $d u$ patient ", de " les soigner et leur donner des conseils sur leur demande d'asile, je les aidais pas en tant que médecin mais beaucoup plus que ça ». Dorreh prenait du temps en consultation, " on pouvait me reprocher de travailler comme un psychologue! ", car le rythme de travail et le nombre de patients ne permettent pas toujours de leur consacrer du temps mais c'était selon ce soignant la solution pour créer une relation de confiance.

75 Comme Dorreh, Françoise a fait cette expérience de se reconstruire professionnellement grâce au sens qu'elle investissait dans ses actes de soin.

« Humainement quand je n'étais pas bien dans ma tête, ça m’a beaucoup aidé. (...)

J'ai eu le sentiment de faire quelque chose, surtout après mon échec et mon burn out, ça m’a vraiment sauvé de cette dépression que j'avais. (...) J’ai retrouvé les valeurs de base (...), la valeur du soin, de faire un pansement (...), des choses qu'on t'a appris et que tu sens naturel. »

76 Ainsi, Dorreh et Françoise ont réinvesti du sens dans ce qu'elles ont fait. Se sentir utile et «faire tout ce qu'on peut » ont permis à Dorreh et Françoise d'inverser la notion de "sale boulot» (Hughes, 1996). C'est le même mécanisme que celui observé par AnneMarie Arborio auprès des aides-soignantes (2012) dont les gestes lors de la toilette sont apparentés à la basse besogne mais qui de leur point de vue contribuent au bien-être des patients. La sociologue les considère comme faisant partie d'un « personnel invisible » qui exerce « un métier exemplaire du care ».

\section{Conclusion}

77 La circulation des médecins afghans prend la forme de l'exil et d'une fuite des cerveaux. Leur intégration - peu commune - à la PASS de Calais crée une relation de type donnant-donnant où professionnels et institution tirent un bénéfice. Les premiers, destitués, se reconnectent au monde médical et peuvent espérer reconquérir leur statut professionnel à terme tandis que l'hôpital peut faire perdurer la médecine des précaires à la PASS et obtenir des effectifs suffisants en mobilisant cette ressource que sont ces soignants exilés. Sur ce «lieu-frontière » où se renforcent année après année les frontières physiques et le rejet des "clandestins ", à l'inverse, au sein de la PASS,

Revue francophone sur la santé et les territoires, Les circulations en santé : des produits, des savoirs, des personnes en mouvement 
des efforts sont réalisés pour tenter d'abolir les frontières symboliques ou de les dépasser en vue de se rapprocher des patients. La circulation des médecins afghans exilés reconfigure les échanges entre soignants en éclairant davantage le pan culturel. Les soignants exilés jouent un rôle de passeurs culturels qui permet de lever les freins aux soins dans la prise en charge adressée aux exilés précaires et d'éclairer les professionnels français sur la singularité de ceux qu'ils ont en face d'eux. Cependant, cette appartenance culturelle qu'ils mobilisent comme un atout peut aussi se retourner contre eux puisque les tensions dans la relation soignants-exilés et patients-exilés sont parfois réactivées. Toutefois, c'est la compassion qui prédomine ces rapports et ce vécu commun qu'est l'exil - même si les soignants exilés semblent plus chanceux que leurs patients - les rapproche et expose davantage ces derniers que des soignants français. Pour autant, ils parviennent à en faire une force. A travers ce sentiment de se sentir utile, ils imprègnent, comme les paramédicaux reclassés, de sens leur travail.

\section{BIBLIOGRAPHIE}

Agier Michel, 2011, Le couloir des exilés, "Etre étranger dans un monde commun", Bellecombeen-Bauges : Editions du croquant.

Arborio Anne-Marie, 2012, Un personnel invisible, Les aides-soignantes à l'hôpital, Paris :

Economica.

Astier Isabelle, Duvoux Nicolas, 2006, La société biographique : une injonction à vivre dignement, Paris : L’Harmattan.

Aubert Nicole et Haroche Claudine, 2011, Les tyrannies de la visibilité, Paris : Eres.

Azria, É., 2013, Le soignant et la standardisation des pratiques médicales. Laennec. Tome 61(3), $32-41$.

Baszanger Isabelle, 1992, La trame de la négociation, Paris : L'harmattan.

Baszanger Isabelle, 1981, Socialisation professionnelle et contrôle social. Le cas des étudiants en médecine futurs généralistes. In: Revue française de sociologie, 22-2. pp. 223-245. https:// www.persee.fr/doc/rfsoc_0035-2969_1981_num_22_2_3411

Baumard Maryline, 2016, « Jungle » de Calais : le démantèlement en chiffres, Le Monde.

Belorgey Nicolas, 2010, L'hôpital sous pression, enquête sur le "nouveau management public", Paris : La découverte.

Bessin Marc, 2003, Le social aux urgences de l'hôpital, île-de-France : Espace éthique.

Bolzman, C., 2014, Exil et errance. Pensée plurielle. 35(1), 43-52.

Bouet Patrick, 2017, Etude comparative des voies de qualification des spécialités médicales.

Conseil national de l'ordre des médecins (Cnom).

Castel Robert, 1995, Les métamorphoses de la question sociale, Paris : Gallimard.

Castra Michel, 2003, Bien Mourir, Paris : Puf.

Revue francophone sur la santé et les territoires, Les circulations en santé : des produits, des savoirs, des personnes en mouvement 
Chamozzi Françoise, 2009, « Risques de l'immigration et déclassement professionnel », Hommes et migrations, 1281 .

Cuttitta P., 2015, La frontière Lampedusa. Mises en intrigue du sécuritaire et de l'humanitaire. Cultures \& Conflits. 99-100, (3), pp. 99-115. doi:10.4000/conflits.19101

Cottereau Victoire, 2015, Les praticiens à diplôme hors Union européenne (PADHUE) en France : Quand les hôpitaux ont recours à des médecins-migrants. Revue francophone sur la santé et les territoires. pp. 1-16.

De Pauw Caroline, 2013. Prise en charge des personnes précaires en médecine générale, Un levier dans la lutte contre les inégalités sociales de santé ? Thèse. Université de Lille. http:// www.theses.fr/2012LIL12014

Dorronsoro Gilles, 1994, Politique et ethnicité en Afghanistan : le cas tadjik. In : CEMOTI, ${ }^{\circ}{ }^{\circ}$, Le Tadjikistan existe-t-il? Destins politiques d'une nation imparfaite. pp. 131-148. doi:10.4000/ cemoti.242

Drexter Armelle, 2008, Le défi du recrutement des médecins à diplômes étrangers dans les hôpitaux publics. Mémoire. EHESP. https://documentation.ehesp.fr/memoires/2008/edh/ drexler.pdf

Fassin Didier et Rechtman Richard, 2007, L'empire du traumatisme, enquête sur la condition de victime, Paris : Flammarion.

Galitzine-Loumpet Alexandra (Cessma Inalco, Liminal) \& Amir Moghani (Cerlom Inalco, Liminal), 2806 2018, ANR LIMINAL JUNGLE - « DJANGALA » - DJEUNGUEL https://liminal.hypotheses.org/ 276

Guenebeaud Camille, 2017, Dans la frontière : migrants et luttes des places dans la ville de Calais, Université de Lille. http://www.theses.fr/2017LIL10079

Hassin Jacques, 1996, L'émergence de l'abord médico social des populations sans toit stable, Université Descartes.

Héran François, 2016, Crise des migrants : Crise migratoire ou crise européenne ?, La journée "Crise des migrants : décentrer le regard", à l'Ined, a été co-organisée par Cris Beauchemin (Ined) et Jean-Luc Primon (Unice-iPOPs).

Hughes Everett C. , 2010, Les honnêtes gens et le sale boulot. Travailler. $\mathrm{N}^{\circ}$ 24. p. 21-34. doi: $10.3917 /$ trav.024.0021

Jonquet Olivier, 2005, L'Afghanistan, Montpellier Hérat : chronique d'une mission. La revue de la faculté de médecine de Nîmes. $\mathrm{N}^{\circ}$ 2, p. 15.

Laacher Smain, 2007, Le Peuple des clandestins, Paris : Calmann-Lévy.

Laacher, Smain, 2005, Éléments pour une sociologie de l'exil. Politix. $\mathrm{N}^{\circ}$ 69, pp. 101-128. doi: $10.3917 /$ pox.069.0101

Laplantine François, 2010, La description ethnographique. L'enquête et ses méthodes, Paris : Colin.

La Voix du Nord, 2017, Long format: Calais, un an après le démantèlement de la "jungle".

Le Breton-Lerouvillois Gwénaëlle , 2014. Les flux migratoires et trajectoires de médecins. Conseil national de l'ordre des médecins (Cnom).

Martuccelli Danilo, 2002 Grammaires de l'individu, Paris : Gallimard. 
Mendy Angele Flora. 2014, La carrière du médecin africain en europe : être médecin avec un diplôme africain au Royaume-uni, en France et en Suisse Swiss. Journal of Sociology. $n{ }^{\circ} 40$, pp. 57-77.

Mercadier Catherine, 2008, Le travail émotionnel des soignants à l'hôpital, Paris: Seli Arslan.

Peneff Jean, 1992, L'hôpital en urgence, Paris : Métailié.

Rodriguez Jacques et Tisserand, 2017, «Calais, « laboratoire » d'une médecine de l'exil ». Hommes et migrations. Paris : $\mathrm{n}^{\circ} 1316$.

Sayad Abdelmalek, 1999, La double absence, Paris : Seuil.

Schepens Florent, 2014, Le recrutement du médecin en unité de soins palliatifs. Sélections, désignation et habilitations. Revue Française de Socio-Économie. $N^{\circ}$ 14. p. 51-70. doi:10.3917/ rfse.014.0051

Smith Stephen, 2018, La Ruée vers l'Europe. La jeune Afrique en route pour le Vieux Continent, Paris : Grasset.

Tisserand et Rodriguez, (2019), « Le toucher comme ressource : soigner les exilés à la frontière ", In Cabral et al. (dir), Le toucher Cabral et al (dir), Le Toucher: Prospections médicales, artistiques et littéraires, Paris : Le Manuscrit.

Vallet Elisabeth, 2014, Borders, Fences and Walls, Ashgate : Border Regions Series.

Younos C., R. Soulimani, N. Seddiqi,O. Et al., 2005, Étude ethnobotanique et historique des tamaris (Tamarix sp.,tamaricaceae) et leurs usages actuels en Afghanistan. Phytothérapie. Vol 3, pp. 248-251. doi:10.1007/s10298-005-0116-8

\section{NOTES}

1. La permanence d'accès aux soins de santé (PASS) est un service de l'hôpital, inscrite dans la loi contre l'exclusion de 1998. La médecine exercée est de type médico-sociale. A Calais, la PASS est dite "fixe", elle possède son propre modulaire situé à quelques mètres du corps principal de l'hôpital.

\section{RÉSUMÉS}

À Calais, "lieu-frontière" (Cuttitta, 2015), l'augmentation du nombre d'exilés a obligé l'hôpital à développer en 2014 son équipe médicale attachée à la permanence d'accès aux soins de santé (PASS), un service qui délivre une médecine hospitalière gratuite pour les patients précaires dépourvus de couverture sociale. Faute de candidatures suffisantes, l'institution a eu recours à des médecins afghans en situation d'exil et qui ont travaillé auprès de soignants français (infirmiers, médecins, psychologues, etc.) Cette expérience a produit des échanges interculturels entre professionnels au sein de la prise en charge des exilés précaires. Les soignants exilés deviennent des passeurs culturels. Ainsi, ils valorisent leur rôle. Pour autant, leur appartenance culturelle qui peut se révéler être un atout et peut aussi se retourner contre eux dans la relation 
avec les patients exilés précaires. Mais plus qu'un lieu de partage interculturel, la PASS constitue un tremplin pour ces soignants exilés en situation de désaffiliation sociale (Castel, 1995) depuis qu'ils ont emprunté les routes de l'exil comme leurs patients et qui souhaitent reconstruire leur identité professionnelle. Ainsi le premier emploi qu'ils acquièrent dans un hôpital français peut leur permettre d'évoluer de statut en statut pour finalement obtenir leurs équivalences relatives à la spécialité qu'ils exerçaient en Afghanistan, ce qui est le cas pour Dorreh, médecin afghan.

Calais is a "border area" (Cuttitta, 2015). In 2014, the increase in the number of exiles forced the hospital to develop its medical team attached to the permanent health care access service (PASS), which provides free hospital medicine for vulnerable patients without social security coverage. The rapport of professionals towards precariousness has been the subject of several sociological studies that have shown that caregivers in hospitals can express disgust towards patients at the margins. In its ancestral form, the hospital welcomes "these people too" (Castel, 1995) but the creation of university hospital centers (CHU) in 1958 has spread somewhat this social dimension in favor of technical prowess and specialties. We will first see how caregivers reclassified exiles as a resource for the hospital to extend medicine to the precarious. This category of professionals comes together and resembles each other because they share the test of medical disconnection that they have known on their professional trajectory. In the absence of sufficient applications, the institution used Afghan doctors who worked with French carers (nurses, doctors, psychologists, etc.). The metamorphoses of the PASS related to the adaptation of the migratory movements thus favor the circulation of the professionals: hospital doctors (French and Afghans), paramedics providing specialized services (pneumology, EHPAD, traumatology, etc.), some of whom are seeking occupational reclassification. There is also a turnover of caregivers that makes the hospital a potential place for the circulation of knowledge and exchanges. This experience produced intercultural exchanges between professionals in the care of precarious exiles. Exiled carers become cultural Interpreters. Because they often speak the language of their patients and they know their culture, the mobilization of these skills informs health professionals and reduces the gap that exists between French health professionals and treated exiles. Their cultural affiliation is an asset but can also be turned against them in the relationship with precarious exiled patients. Being Afghan presents advantages and may also pose difficulties in the form of reluctance, albeit rarely, on the part of patients. There is a risk of mirror effect because carers and caregivers share a common experience, which can lead caregivers to redouble their efforts at work. However, it is compassion that predominates these relationships and the common experience of exile - even if the exiled caregivers seem more fortunate than their patients - brings them closer and exposes them more than French health care providers. Through this feeling of being useful, they permeate, like the reclassified paramedics, the meaning of their work. But more than a place of intercultural sharing, PASS is a springboard for these exiled carers in a situation of social disaffiliation (Castel, 1995), since they have taken the same roads of exile as their patients and who wish to rebuild their professional identity. Exile is a shock, a crack since "tearing out the country" (Laacher, 2005) implies a situation of radical rupture. Caregivers had to give up their social status to blend in with those who find themselves on the roads; they become like other exiles, "supernumeraries" (Castel, 1995). Thus, the first job they acquire in a French hospital may allow them to evolve from status to status and finally obtain their equivalence in terms of the specialty they were practicing in Afghanistan.

\section{INDEX}

Mots-clés : frontière, circulation, médecine humanitaire Index géographique : Afghanistan 


\section{AUTEUR}

\section{CHLOÉ TISSERAND}

Doctorante au centre de recherche "Individus, épreuves et sociétés" (CeRIES) à l'Université de Lille. Fellow à l'Institut convergences migrations (ICM) à Paris 